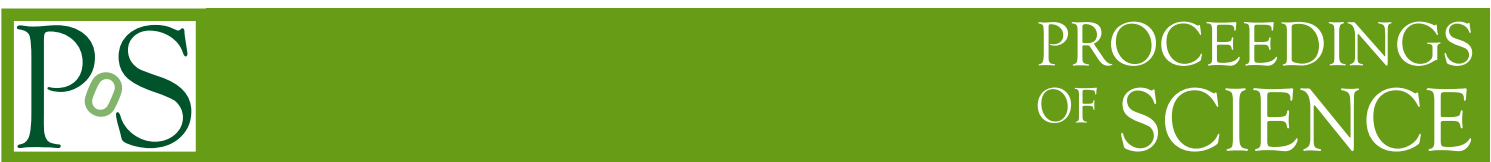

\title{
The effects of accretion luminosity from Pop III protostars
}

\author{
Rowan J. Smith*1, Paul C. Clark ${ }^{1}$, Simon C. O. Glover ${ }^{1}$, Thomas Greif ${ }^{2}$ and Ralf S. \\ Klessen ${ }^{1}$ \\ ${ }^{1}$ Zentrum für Astronomie der Universität Heidelberg, Institut für Theoretische Astrophysik, \\ Albert-Ueberle-Str. 2, 69120 Heidelberg, Germany \\ ${ }^{2}$ Max-Planck-Institut für Astrophysik, Karl-Schwarzschild-Str. 1, 85740 Garching bei München, \\ Germany E-mail: rowan@ita.uni-heidelberg.de
}

\begin{abstract}
We introduce a prescription for accretion luminosity feedback into an smooth particle hydrodanamic simulation of five primordial minihalos generated from cosmological initial conditions. We find that accretion luminosity delays fragmentation within the halos, but does not prevent it. In halos that fragment slowly the accretion luminosity reduces the number of fragments that are formed before ionisation from the protostars becomes effective, however halos that fragment rapidly quickly become dominated by dynamical processes and the effect of accretion luminosity becomes negligible. Generally the fragmentation found in the halos is highly dependent on the initial conditions. Accretion luminosity does not substantially affect the accretion rates experienced by the protostars, and is far less important than dynamical interactions which can halt accretion entirely due to ejections.
\end{abstract}

Cosmic Radiation Fields: Sources in the early Universe - CRF2010,

November 9-12, 2010

Desy Germany

\footnotetext{
* Speaker.
} 


\section{Motivation}

Over the last decade the picture of star formation in the primordial universe was that of single massive stars that were the sole inhabiters of the first dark matter minihalos formed after the big bang. This was the early finding of the numerical models of $[1,2]$, and was later reinforced by the findings of [3]. From this [4] used detailed stellar modelling to estimate a final mass of the primordial star and found that masses in excess of $100 \mathrm{M}_{\odot}$ could be produced.

However the above mentioned simulations shared the problem of being unable to proceed beyond the first stage of collapse due to the numerical difficulty of following the evolution of high density gas, where the numerical time step becomes prohibitively small. More recent work, which follows the collapse beyond the formation of the first protostellar core, has cast doubt on the isolated picture of Population III star formation, suggesting that they may have been members of binaries, multiples, or even small-N clusters [5-9]. In the most recent work [10] find that the individual disks around population III stars are prone to fragmentation and are likely to fragment into higherorder multiples. This result is confirmed by [11] using the AREPO [12] method in multiple halos with sink particles as small as the actual protostars. The case for fragmentation therefore seems robust, as it has been found by multiple authors using a variety of techniques, and if true would have important implications for our understanding of cosmology and the early universe

Given the importance of these results, it is important to consider whether there are any mechanisms that could suppress fragmentation. Ionisation from the protostar is the most likely mechanism to stop further fragmentation, but this only becomes significant once the protostar starts contracting to the main sequence [13]. One of the few processes that would act against fragmentation before this epoch, is accretion luminosity feedback from the forming protostars. We have introduced this in [10] to study disk fragmentation. [10] finds that accretion luminosity does indeed change the disk evolution, but that it cannot ultimately prevent fragmentation. The feedback is able to support the inner regions of the disk, which were previously unstable, against fragmentation, but the outer regions still fragment, albeit after a longer time period. We now seek to address a new question. The optimal time for fragmentation within a minihalo is the first few thousand years before the first protostar approaches the main sequence and ionises its surrounding gas. In this contribution we aim to capture the full evolution of the halo during this regime to determine how much fragmentation can occur, and to what effect radiation affects the fragmentation.

\section{Method}

We perform our calculations using the SPH code GADGET 2 [14]. We have substantially modified this code to include a full time-dependent chemical network, details of which can be found in the appendix of [8]. We include heating from the accretion luminosity as an additional heating term when solving the ordinary differential equations that govern the chemical and thermodynamic evolution of the gas.

The accretion luminosity is calculated from the standard equation,

$$
L_{a c c}=\frac{G M_{*} \dot{M}}{R_{*}}
$$


where $\dot{M}$ is the accretion rate of the protostar and $R_{*}$ is the stellar radius. We make the assumption that the gas is optically thin to the emitted radiation which ensures we are overestimating the effects of the accretion luminosity to obtain an upper limit on the feedback effects. The heating rate for the gas will then become

$$
\Gamma_{a c c}=\rho_{g} \kappa_{P}\left(\frac{L_{a c c}}{4 \pi r^{2}}\right) \operatorname{erg~g}^{-1} \mathrm{~s}^{-1}
$$

where $\rho_{g}$ is the gas density, $\kappa_{P}$ is the Plank mean opacity and $r$ is the distance of the gas from the source. We calculate the Planck mean opacity of the gas by interpolating from the tables of [15] which includes contributions from deuterium and lithium in the gas in addition to hydrogen and helium. To accurately calculate the accretion luminosity, both the accretion rate and the stellar radius need to be known. We achieve this by using sink particles [16] to model the protostars and record their growth in mass throughout the simulation to calculate the accretion rate. Accurately finding the stellar radii would require the implementation of detailed stellar evolution models within our hydrodynamic simulation which is beyond current computational resources. Instead we used the models of [4] to derive a simple power law approximation of the stellar radius. For a full description of this procedure see Smith et al. 2011 submitted.

In order to capture the true properties of the first minihalos within which primordial stars form, we use the cosmological simulations of [11] as initial conditions. [11] presented simulations of primordial minihalos that strongly fragmented. These simulations made use of the novel moving mesh code AREPO [12] to fully follow and resolve the formation of five minihalos from fully cosmological simulations. All of the halos of [11] form multiple protostars with a range of masses. For this work we resimulate the central two parsecs of these simulations with feedback.

\section{Results}

Figure 1 shows a column density projection of the central regions one of our halos. In all cases a disk-like structure is formed due to the inability of the halo to transfer angular momentum outwards quickly enough during collapse. While the central region is disk-like it is more extended and irregular than a true disk. In all cases this region fragments. Generally several fragments form almost simultaneously as the conditions for fragmentation are reached at multiple locations within the disk. Halos 1 and 5 fragment vigorously, whereas halos 2 and 3 fragment more slowly. Halo 4 is the case that is most affected by accretion luminosity and is the only one which substantially differs from the original simulations presented in [11]

Table 1 shows the number of fragments formed in each halo when the most massive protostar is first $10 \mathrm{M}_{\odot}$. [13] find that ionising feedback does not become effective until the star is older than its Kelvin-Helmholtz time and is contracting towards the main sequence. For their fiducial model this equates to a mass of around $30 \mathrm{M}_{\odot}$ for a rotating protostar. However the accretion rate for the most massive object is typically only a few $10^{-3} \mathrm{M}_{\odot} \mathrm{yr}^{-1}$ when the protostar has reached 10 $\mathrm{M}_{\odot}$ in our minihalos, whereas in the fiducial [13] models the accretion rate is $10^{-2} \mathrm{M}_{\odot} \mathrm{yr}^{-1}$ for a 10 $\mathrm{M}_{\odot}$ protostar. Since the Kelvin-Helmholtz contraction stage commences earlier with a lower accretion rate, we estimate that ionisation feedback will become important for our minihalos when the most massive star is between $10-15 \mathrm{M}_{\odot}$. Beyond this point our simulations become unreliable.

Perhaps the most striking feature of Table 1 is the amount of variability between the halos. Halo 5 forms a star greater than $10 \mathrm{M}_{\odot}$ after only $600 \mathrm{yr}$ without feedback, whereas Halo 3 takes 


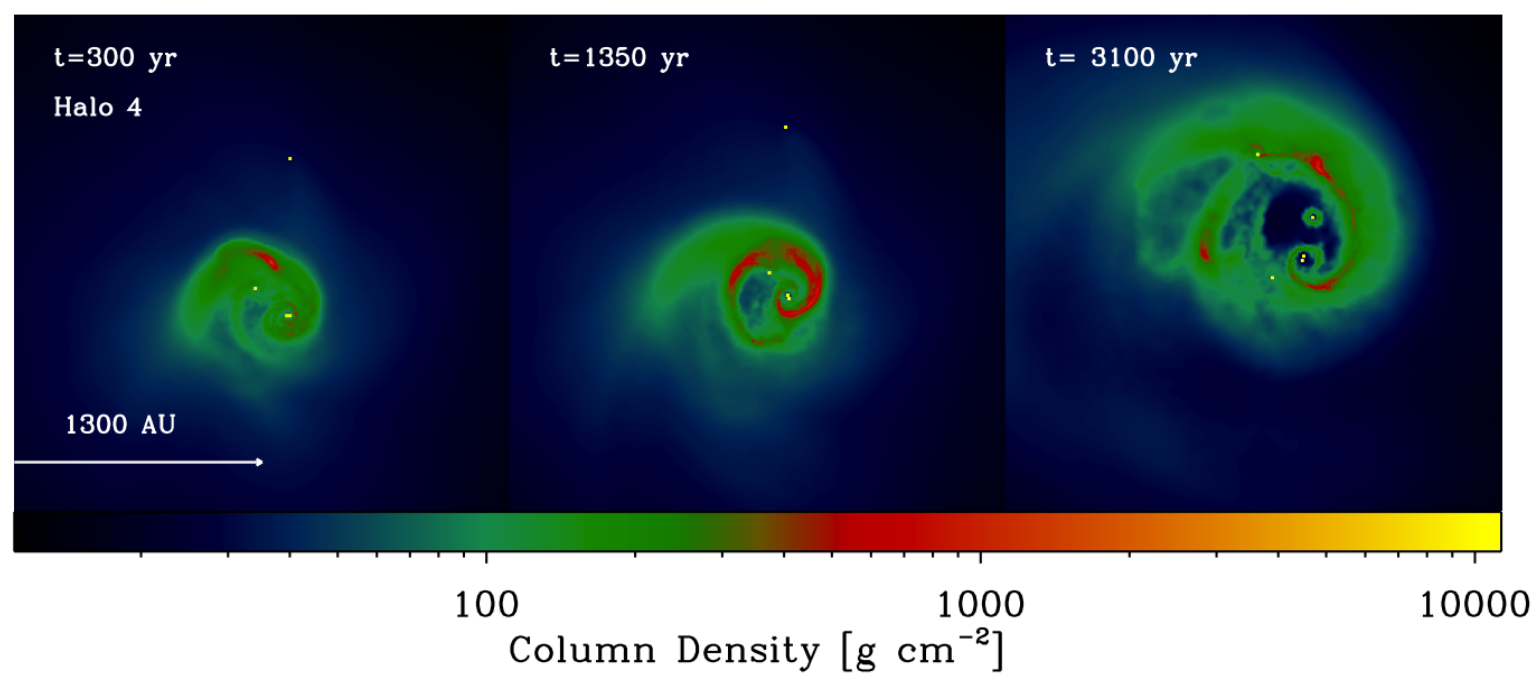

Figure 1: Column density projection of fragmentation seen in the central 1300AU in the first few thousand years for Halo 4. Sink particles are denoted by yellow squares. Despite the presence of accretion luminosity heating there is still fragmentation.

Table 1: The number of stars and time when the most massive star in the minihalo had a mass of $10 \mathrm{M}_{\odot}$. The effects of ionising radiation are estimated to become effective between 10 and $15 \mathrm{M}_{\odot}$ which is likely to suppress further fragmentation. There is significant inter-halo variation both in the number of fragments and the duration in which accretion luminosity is the dominant feedback mechanism. For equivalent halos the one which forms the massive star most quickly has the least fragmentation.

\begin{tabular}{cllll}
\hline \hline Halo & Ref. & \multicolumn{3}{l}{ Feedback } \\
\hline & No. & Time [yr] & No. & Time [yr] \\
\hline 1 & 10 & 1,520 & 10 & 2,520 \\
2 & 10 & 7,640 & 7 & 4,490 \\
3 & $5+$ & 9,430 & 5 & 5,140 \\
4 & 17 & 7,320 & 5 & 1,010 \\
5 & 7 & 604 & 18 & 1,440 \\
\hline
\end{tabular}

over $10,000 \mathrm{yr}$ to do so. In the time to taken to reach $10 \mathrm{M}_{\odot}$ the number of fragments varied between 5 and 17. Therefore inter-halo variability is at least as important an effect as accretion luminosity feedback. The variability of the halos can be traced back to their chemical evolution during their collapse. [11] found that in two of the halos considered here (halos 2 and 3) there was significant HD cooling which allowed the gas to cool to temperatures as low as $100 \mathrm{~K}[17,18]$. When the gas was reheated by compression in the final stages of the collapse this smoothed out some of the small scale structure, resulting in less fragmentation. In the remaining three halos HD cooling was not activated and so temperatures only as low as $200 \mathrm{~K}$ were obtained via $\mathrm{H}_{2}$ cooling, in this case the subsequent reheating was less violent and more small scale structure was retained.

Figure 2 shows the evolution of fragmentation within the halos. Halos 1 and 5 fragment rapidly, meaning they quickly become dominated by chaotic dynamical interactions and the refer- 

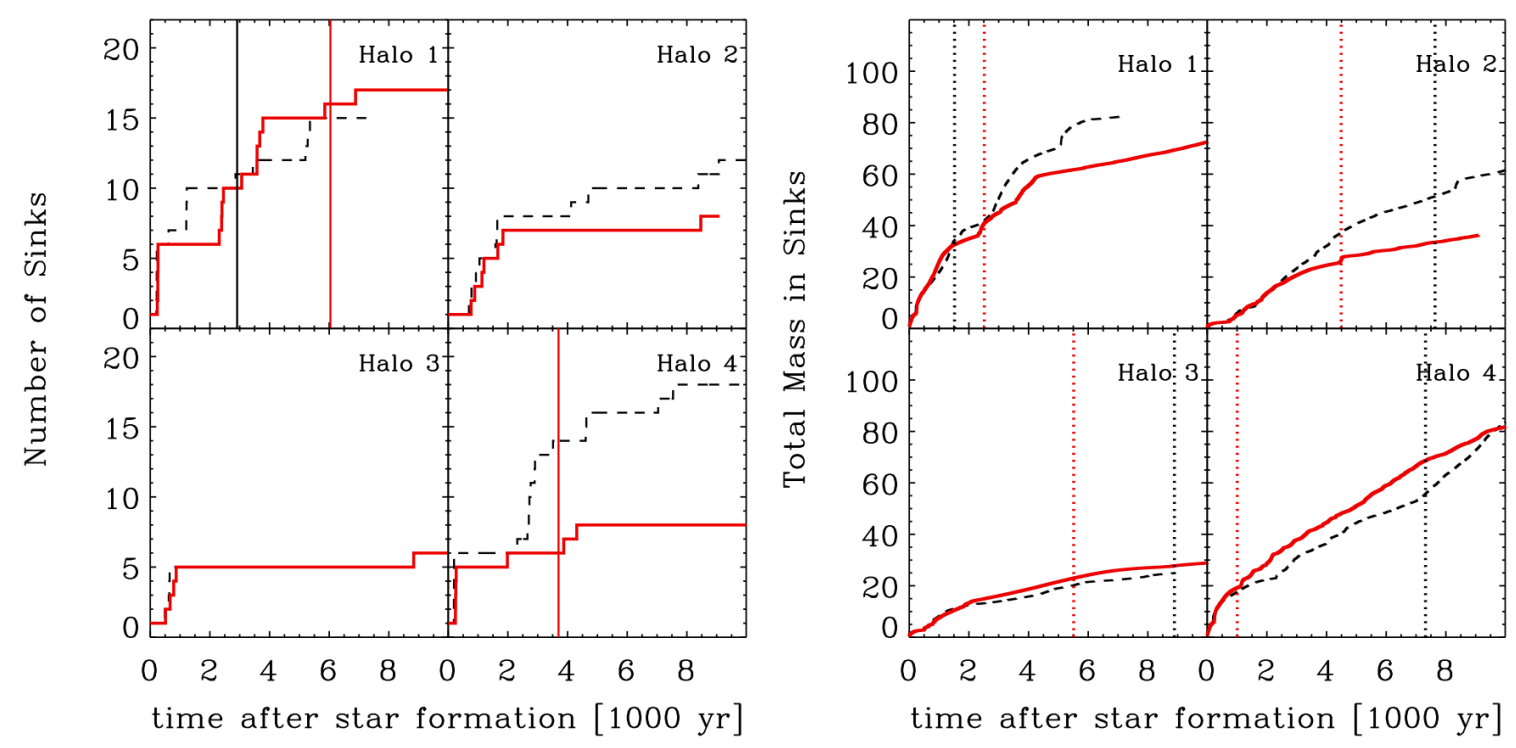

Figure 2: The combined properties of the sinks formed in each halo plotted against the time after the first fragment forms. The solid red line shows the case with feedback and the black dotted line the case without. The vertical solid red and black lines in panel one show the point at which a star reaches $15 \mathrm{M}_{\odot}$ and the dotted vertical lines in panel two show when $10 \mathrm{M}_{\odot}$ has been reached.

ence and feedback cases are no longer equivalent. It is found that the halo which takes the longest to reach the ionising mass undergoes the most fragmentation, simply by virtue of having longer to fragment. Dynamical interactions are therefore as important as accretion luminosity effects in the rapidly fragmenting case. For example in the run of Halo 1 with feedback, there was a dynamical interaction which ejected the most massive star before it could reach $10-15 \mathrm{M}_{\odot}$, consequently there was more time for fragmentation until one of the originally lower mass objects reached this mass. Sufficient sinks are formed within Halo 5 that chaotic N-body interactions mean the feedback and reference cases diverge so quickly that no clear conclusions can be made about the feedback and so its evolution is not shown in Figure 2.

Halos 2 and 3 are more straightforward as these halos fragment and accrete material less vigorously. As fewer fragments are formed, there is less competition to accrete the gas, which allows the first fragments to grow in mass and substantially heat their surroundings. This delays when the fragmentation occurs in the feedback case compared to the reference case. In Figure 2 the fragmentation generally follows the that of the reference case, and in some cases the delay can be as great as a thousand years. This was also true in Halo 1 until the chaotic dynamics made the runs diverge after a thousand years or so. The delaying of fragmentation seems to be the major consequence of accretion luminosity feedback. This was also the conclusion reached in the protostellar disk studied in [10]. Although feedback has not prevented fragmentation, this delay has meant that there are fewer fragments when ionising feedback becomes effective, so in total accretion feedback has reduced the number of stars formed.

Halo 4 is the case in which the maximal effect of the feedback was seen. Like Halos 1 and 5, HD cooling was not activated and significant fragmentation occurred in the reference case. How- 
ever, with feedback the number of fragments formed before $10 \mathrm{M}_{\odot}$ was reduced from 17 fragments to 7. In this case there was a sufficient delay between the second bout of fragmentation after the disk was first unstable that the sinks were massive enough to produce a significant luminosity. This effect was enhanced by the geometry of the resulting system ( as seen in Figure 1 ) with all the sink particles remained within the central disk-like region where they could heat the dense gas.

The total mass that goes into sink particles is also shown in Figure 2 and is found is broadly unaffected by the feedback. This is due to the dense gas being heated, but not unbound. Consequently it can still be accreted by the protostars.

\section{Conclusions}

We have introduced a prescription for heating from accretion luminosity into a re-simulation of five minihalos from cosmological initial conditions. We followed the evolution of these halos with and without feedback up until the point at which ionisation feedback would become significant and found that,

1. Accretion luminosity delays fragmentation but cannot prevent it.

2. The intrinsic variation in halo properties due to differences in their formation history, generally has a larger effect on the number of fragments formed, than accretion luminosity.

3. Halos in which a large number of fragments form rapidly are dominated by dynamical effects. It is only in more slowly fragmenting cases which form fewer fragments, that accretion luminosity becomes effective.

\section{References}

[1] T. Abel, G. L. Bryan, and M. L. Norman, The Formation and Fragmentation of Primordial Molecular Clouds, ApJ 540 (Sept., 2000) 39-44, [arXiv : astro-ph / 0002135$].$

[2] T. Abel, G. L. Bryan, and M. L. Norman, The Formation of the First Star in the Universe, Science 295 (Jan., 2002) 93-98, [arXiv: astro-ph/0112088].

[3] N. Yoshida, K. Omukai, and L. Hernquist, Protostar Formation in the Early Universe, Science 321 (Aug., 2008) 669-, [0807.4928].

[4] K. Omukai and F. Palla, Formation of the First Stars by Accretion, ApJ 589 (June, 2003) 677-687, [arXiv:astro-ph/0302345].

[5] M. N. Machida, K. Omukai, T. Matsumoto, and S. Inutsuka, Conditions for the Formation of First-Star Binaries, ApJ 677 (Apr., 2008) 813-827, [0711.0069].

[6] P. C. Clark, S. C. O. Glover, and R. S. Klessen, The First Stellar Cluster, ApJ 672 (Jan., 2008) 757-764.

[7] M. J. Turk, T. Abel, and B. O'Shea, The Formation of Population III Binaries from Cosmological Initial Conditions, Science 325 (July, 2009) 601-, [0 907.2919$].$ 
[8] P. C. Clark, S. C. O. Glover, R. S. Klessen, and V. Bromm, Gravitational Fragmentation in Turbulent Primordial Gas and the Initial Mass Function of Population III Stars, ApJ 727 (Feb., 2011) 110-+, [1006.1508].

[9] A. Stacy, T. H. Greif, and V. Bromm, The first stars: formation of binaries and small multiple systems, MNRAS 403 (Mar., 2010) 45-60, [0 908 . 0712].

[10] P. C. Clark, S. C. O. Glover, R. J. Smith, T. H. Greif, R. S. Klessen, and V. Bromm, The Formation and Fragmentation of Disks around Primordial Protostars, Science online early http://www.sciencemag.org/ content/early/2011/02/02/science.1198027 (2011).

[11] T. Greif, V. Springel, S. White, S. Glover, P. Clark, R. Smith, R. Klessen, and V. Bromm, Simulations on a Moving Mesh: The Clustered Formation of Population III Protostars, ArXiv e-prints 1101.5491 (Jan., 2011) [1101.5491].

[12] V. Springel, E pur si muove: Galilean-invariant cosmological hydrodynamical simulations on a moving mesh, MNRAS 401 (Jan., 2010) 791-851, [0901 . 4107].

[13] J. C. Tan and C. F. McKee, The Formation of the First Stars. I. Mass Infall Rates, Accretion Disk Structure, and Protostellar Evolution, ApJ 603 (Mar., 2004) 383-400,

[arXiv:astro-ph/0307414].

[14] V. Springel, The cosmological simulation code GADGET-2, MNRAS 364 (Dec., 2005) 1105-1134, [arXiv:astro-ph/0505010].

[15] M. Mayer and W. J. Duschl, Rosseland and Planck mean opacities for primordial matter, MNRAS 358 (Apr., 2005) 614-631, [arXiv: astro-ph/ 0411613$].$

[16] M. R. Bate, I. A. Bonnell, and N. M. Price, Modelling accretion in protobinary systems, MNRAS 277 (Nov., 1995) 362-376, [arXiv:astro-ph/9510149].

[17] E. Ripamonti, The role of HD cooling in primordial star formation, MNRAS 376 (Apr., 2007) 709-718, [arXiv:astro-ph/0701148].

[18] I. D. McGreer and G. L. Bryan, The Impact of HD Cooling on the Formation of the First Stars, ApJ 685 (Sept., 2008) 8-20, [0 802 .3918]. 